\title{
Non-Blinking Single-Photon Generation with Anisotropic Colloidal Nanocrystals: Towards Room-Temperature, Efficient, Colloidal Quantum Sources
}

\author{
Ferruccio Pisanello,* Godefroy Leménager, Luigi Martiradonna, Luigi Carbone, \\ Stefano Vezzoli, Pascal Desfonds, Pantaleo Davide Cozzoli, Jean-Pierre Hermier, \\ Elisabeth Giacobino, Roberto Cingolani, Massimo De Vittorio, and Alberto Bramati
}

It is well-known that colloidal semiconductor nanocrystals, ${ }^{[1]}$ i.e., wet-chemically synthesized quantum dots, can emit nonclassical light also at room temperature. ${ }^{[2]}$ However, in the field of quantum sources many other nano-materials are considered as state-of-the-art alternatives to nanocrystals, such as, color centers in diamond nanocrystals, ${ }^{[3,4]}$ Stransky-Krastanov quantum dots, ${ }^{[5]}$ and certain organic molecules. ${ }^{[6]}$ The main

\author{
Dr. F. Pisanello, Dr. L. Martiradonna, \\ Prof. M. De Vittorio \\ Istituto Italiano di Tecnologia (IIT) \\ Center for Bio-Molecular Nanotechnologies \\ Via Barsanti sn, 73010 Arnesano (Lecce), Italy \\ E-mail: ferruccio.pisanello@iit.it \\ Dr. F. Pisanello, Dr. G. Leménager, Dr. S. Vezzoli, \\ Dr. P. Desfonds, Prof. E. Giacobino, Prof. A. Bramati \\ Laboratoire Kastler Brossel \\ Université Pierre et Marie Curie \\ CNRS UMR 8552, Ecole Normale Supérieure, \\ 4 place Jussieu, 75252 Paris Cedex 05, France \\ Dr. F. Pisanello \\ Center for Neuroscience and Cognitive Systems @UNITN \\ Istituto Italiano di Tecnologia \\ corso Bettini 31, 38068 Rovereto (TN), Italy \\ Dr. L. Carbone, Dr. P. D. Cozzoli, Prof. M. De Vittorio \\ National Nanotechnology Laboratory (NNL)- CNR Istituto Nanoscienze, \\ c/o Distretto Tecnologico, via per Arnesano km 5, 73100 Lecce, Italy \\ Dr. P. D. Cozzoli \\ Dipartimento di Matematica e Fisica "E. De Giorgi" - Università del \\ Salento, via per Arnesano, 73100 Lecce, Italy \\ Dr. J.-P. Hermier \\ Groupe d'étude de la Matière Condensée \\ CNRS UMR8635, Université de Versailles \\ Saint-Quentin-en-Yvelines \\ 45 avenue des Etats-Unis, 78035 Versailles Cedex, France \\ Dr. J.-P. Hermier \\ Institut Universitaire de France \\ 103, Boulevard Saint-Michel, F-75005, Paris, France \\ Prof. R. Cingolani \\ Istituto Italiano di Tecnologia (IIT) \\ Via Morego 30, 16163 Genova, Italy \\ Prof. M. De Vittorio \\ Dipartimento di Ingegneria dell'Innovazione - Università del \\ Salento, via per Monteroni, 73100 Lecce, Italy \\ Istituto Italiano di Tecnologia (IIT) \\ Center for Bio-Molecular Nanotechnologies \\ Via Barsanti sn, 73010 Arnesano (Lecce), Italy
}

DOI: 10.1002/adma.201203171 reason for this can be found in the continuous competition between radiative and non-radiative recombination channels that, in nanocrystals, generates photoluminescence (PL) fluctuations between on and off periods known as blinking. ${ }^{[7]}$ Several reports have suggested nanocrystal shell engineering as a suitable strategy to control recombination paths, producing almost non-blinking nanocrystals, ${ }^{[8-10]}$ although accompanied by undesired increasing of multi-excitonic emission probability. ${ }^{[11-13]}$ Here we show that, in dot-in-rod (DR) core/shell nanoparticles with a spherical CdSe core surrounded by a rod-like CdS shell (see sketch in Figure 1), ${ }^{[14-16]}$ two different degrees of freedom in shell engineering can be exploited to obtain non-blinking single-photon generation.

The physical explanation of PL random switching between on and off periods is still under debate ${ }^{[17-19]}$ and different mechanisms have been proposed to account for the peculiar photophysical properties of off states (see Supporting Information (SI) for a discussion on this subject). The most used model assigns this intermittency to an interchange between neutral and ionized states of the whole nanoparticle. ${ }^{[19,20]}$ When the nanocrystal is in the so-called trion configuration (one exciton plus another charge), ${ }^{[19-26]}$ the additional charge can catch the exciton energy otherwise emitted radiatively, giving rise to a non-radiative Auger recombination process. This energy transfer typically occurs at rates several orders of magnitude faster than radiative recombination, ${ }^{[27-29]}$ thus quenching the PL. ${ }^{[19,20]}$ In order to reduce the environmental sensitivity and thus the ionization probability, the single nanoparticle is usually enclosed in a crystalline shell of another material, thus obtaining a nano-heterostructured nanocrystal (NC) in the core/shell configuration. Moreover, besides acting on the probability to undergo ionization processes, the presence of a shell also modifies carriers confinement and their mutual interaction strengths. Indeed, by thickening the shell ${ }^{[8,9]}$ or inducing smooth variations of confinement potential at core/shell interface, ${ }^{[10]}$ Auger recombination rates in ionized particles can be modified and blinking suppressed thereof. ${ }^{[8-10]}$ Nevertheless, as shell tailoring can work straightforwardly on charge carrier confinement mechanism, NCs effectiveness as single-photon emitters can be altered. ${ }^{[11,12]}$ Indeed, also multi-photon emission probability is ruled by a non-radiative and confinementdependent Auger process. ${ }^{[11,28,30,31]}$ For instance, an increase of shell size in spherical CdSe/CdS nanocrystals also decreases Auger interactions among multiple excitons, ${ }^{[32,33]}$ leading to 
(a)
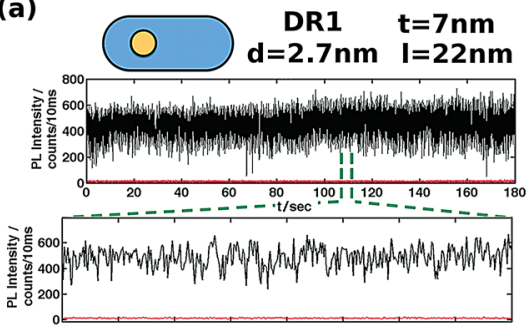

(c)
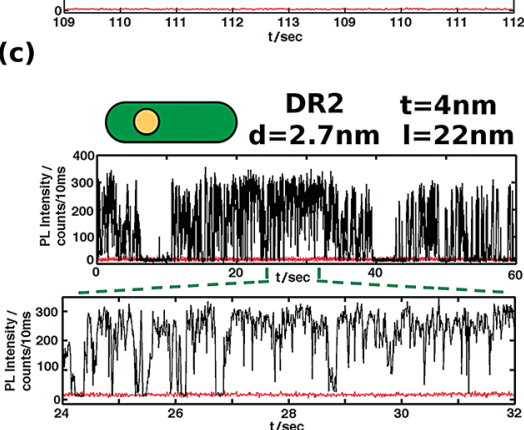

28 (b)

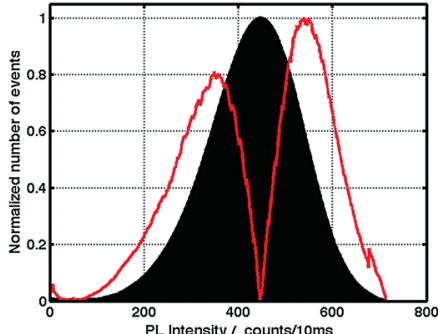

(d)

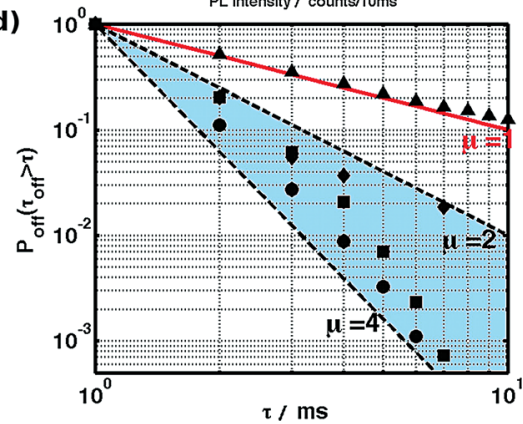

Figure 1. DRs PL evolution. a) Typical PL intensity with a temporal zoom for a thick-shell DR (sample DR1). Red lines represent the noise counts. Binning time is $10 \mathrm{~ms}$. b) Intensity histogram of the time trace reported in panel (a). Red line is the absolute value of the first order derivative of the histogram: the difference between the two maxima shows that the slope of the histogram is not the same on the two sides of its maximum. Binning time is $10 \mathrm{~ms}$. c) Typical PL intensity with a temporal zoom for a thin-shell DR (sample DR2), showing pronounced blinking. Red lines represent the noise counts. Binning time is $10 \mathrm{~ms}$. d) Typical cumulative distribution of off-period durations: circles, diamonds, and squares are measured values for thick-shell DRs (DR1, DR3, and DR4, respectively), while triangles show the values obtained for sample DR2. All measurements performed on samples belonging to DR1, DR3, and DR4 typologies fall within the light-blue area delimited by two dashed black lines, corresponding to power law distributions with $\mu=2$ and $\mu=4$. Red continuous line represents the limit above which the distribution becomes heavy-tailed. Binning time is $1 \mathrm{~ms}$.

biexciton emission with near unity quantum yield. ${ }^{[11]}$ This recombination path, effective for processes like multi-photon emission, ${ }^{[11,12,29,31]}$ high optical gain ${ }^{[34]}$ or charge multiplication, ${ }^{[28,35]}$ is instead detrimental for the development of singlephoton sources, ${ }^{[11,12,29,31]}$ and must be carefully addressed.

Despite these evidences suggest a strong tie between blinking and multiphoton emission, Park and coworkers ${ }^{[11]}$ have recently shown that even non-blinking NCs nominally identical in size and shape may exhibit very different behaviors in terms of single-photon emission properties. The large heterogeneity in biexciton quantum efficiency obtained in ref. ${ }^{[11]}$ suggests, indeed, that blinking and single-photon emission are not necessarily intercorrelated. ${ }^{[11,13,26]}$ However, to the best of our knowledge, a technique to separately address these two processes in a controlled fashion has not been demonstrated till now. Here we show that the independent tuning of shell length and thickness of high-quality colloidal CdSe/CdS core/shell DRs (see sketch Figure 1) results in a better control of photon statistics, allowing to obtain room-temperature non-blinking single-photon generation.

The peculiarity of CdSe/CdS DRs relies in their electronic structure, designed as quasi type-II: although core/shell bands alignment would imply the localization of both carriers into the core, the very small offset of the conduction bands allows electrons wavefunction to be spread on the entire NC. ${ }^{[9,32,36,37]}$

Because of that, shell energy landscape strongly influences the electrons wavefunction $\left(\Psi_{\mathrm{e}}\right)$ while preserving holes confinement. Colloidal CdSe/CdS DRs have already been demonstrated to show remarkable single-photon polarization properties, ${ }^{[38,39]}$ high room-temperature PL quantum efficiency ${ }^{[15,40]}$ and interesting compatibility with planar nanofabrication technologies. ${ }^{[41-45]}$ The DRs investigated in this work have been produced by a wet-chemical seeded-growth approach, ${ }^{[15]}$ a synthetic procedure that allows a precise control of the heterostructure geometrical parameters. ${ }^{[16,46]}$ Very importantly, the synthesis protocol has been modified in order to thicken the shell size, resulting in a total DR thickness of $t \approx 7 \mathrm{~nm}$ against a thinner standard width of $t \approx 4 \mathrm{~nm}$ on

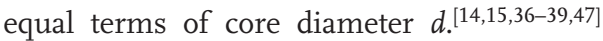
Our approach, involving high growth temperatures $\left(380{ }^{\circ} \mathrm{C}\right)$ and long reaction times (20-30 min), fosters CdS shell to thicken in the volume section around the core and very reasonably promotes the formation of $\mathrm{Cd}, \mathrm{S}$ and Se alloyed layers at the core/shell interface. ${ }^{[32]}$ The latter contributes to diminish the crystal strain between core and shell, and the possible development of planar crystal defects thereof. Remarkably, it also contributes to avoid sharp variations of carriers confinement potential. ${ }^{[10,15,16,32]}$

All optical investigations presented in this work are performed at single particle level by means of a confocal microscope, equipped with a picosecond-pulsed laser diode exciting on average $\langle N\rangle$ electron-hole (e-h) pairs per pulse. We have synthesized and analyzed five different DRs samples, hereafter called DR1, DR2, DR3, DR4, and DR5, all having similar central emission wavelengths $\left(\lambda_{\mathrm{DR} 1}=605 \mathrm{~nm} \pm 1 \mathrm{~nm}, \lambda_{\mathrm{DR} 2}=604 \mathrm{~nm} \pm 1 \mathrm{~nm}, \lambda_{\mathrm{DR} 3}=\right.$ $601 \mathrm{~nm} \pm 1 \mathrm{~nm}, \lambda_{\mathrm{DR} 4}=595 \mathrm{~nm} \pm 1 \mathrm{~nm}, \lambda_{\mathrm{DR} 5}=579 \mathrm{~nm} \pm 1 \mathrm{~nm}$, see SI for full absorption and emission spectra). DR1, DR3, and DR4 present core diameters of $d_{\mathrm{DR} 1}=d_{\mathrm{DR} 3}=d_{\mathrm{DR} 4} \approx 2.7 \mathrm{~nm}$, pronounced shell thickness around the core resulting in total width $t_{\mathrm{DR} 1}=t_{\mathrm{DR} 3}=t_{\mathrm{DR} 4} \approx 7 \mathrm{~nm}$ and shell lengths $l_{\mathrm{DR} 1} \approx 22 \mathrm{~nm}$, $l_{\mathrm{DR} 3} \approx 35 \mathrm{~nm}$, and $l_{\mathrm{DR} 4} \approx 58 \mathrm{~nm}$, respectively (see Figure $1 \mathrm{~S}$ ). Sample DR2 is the counterpart of sample DR1 with standard shell thickness around the core $\left(d_{\mathrm{DR} 1}=d_{\mathrm{DR} 2} \approx 2.7 \mathrm{~nm}, l_{\mathrm{DR} 1}=\right.$ $l_{\mathrm{DR} 2} \approx 22 \mathrm{~nm}$ and $\left.t_{\mathrm{DR} 2} \approx 4 \mathrm{~nm}\right)$. DR5 is instead a large-core particle, having $d_{\mathrm{DR} 5} \approx 4.2 \mathrm{~nm}, l_{\mathrm{DR} 5} \approx 42 \mathrm{~nm}$, and $t_{\mathrm{DR} 5} \approx 7.5 \mathrm{~nm}$.

In order to demonstrate that blinking suppression and single-photon generation can be obtained by acting on DRs geometrical configuration, we first show that blinking-free highintensity emission from single DRs can be induced by thickening the CdS shell. Next, by using time-resolved and second order autocorrelation function $\left[g^{(2)}(\tau)\right]$ analyses, we assess the single-photon emission properties as a function of DRs length. Hence, we show both abatement of blinking and the possibility to obtain single-photon emission even at high excitation density when the emitter is in the saturation regime. 
A PL time trace of a thick isolated DR (sample DR1) and its corresponding photon distribution are displayed in Figure 1a,b. During the time window of $180 \mathrm{sec}$, the DR is almost always emitting, with an average photon rate of $\sim 5 \times 10^{4}$ photons $/ \mathrm{sec}$. Based on the experimental setup collection efficiency $(\sim 2.56 \%$, see SI), we can assess that, under a $2.5 \mathrm{MHz}$ pulsed excitation, the number of photons emitted by a nanoparticle is $\sim 1.95$ Mphotons/sec ( 0.32 Mphotons/sec in the first lens), thus resulting in a PL overall efficiency of $\sim 78 \%$. As control experiment, in Figure 1c we report a typical time trace of sample DR2 having the same core diameter and shell length but thinner shell: a pronounced blinking is shown.

With the purpose to quantify the relevance of fluctuations in thick DRs PL intensity, we monitored the probability distribution of the off periods, $P_{\text {off }}\left(\tau_{\text {off }}>\tau\right)$. Here we consider as off all emitting periods with PL intensity lower than a threshold (th) fixed as 5-times the noise floor (time traces at $1 \mathrm{~ms}$ binning considered for this statistical analysis are reported in Figure S6 of the SI). For several types of luminescent molecules as well as for NCs, it has been demonstrated that $P_{\text {off }}\left(\tau_{\text {off }}>\tau\right)$ can be represented to a first approximation by a power law distribution: ${ }^{[21]}$ $P_{\text {off }}\left(\tau_{\text {off }}>\tau\right) \approx 1 / \tau^{\mu}$. From a statistical point of view, $\mu$ plays a key role: the higher the parameter $\mu$, the lower the probability to have long off periods. As demonstrated for spherical CdSe/ZnS core/shell NCs showing pronounced blinking, values included in the range $0.5<\mu<1$ are associated with Lévy statistics, leading to a non-negligible probability to have off periods as long as the observation time. In contrast, it has been demonstrated that giant shell CdSe/CdS spherical NCs show $\mu$ values higher than 2 , resulting in strong blinking suppression. ${ }^{[9]}$ In the latter case $P_{\text {off }}\left(\tau_{\text {off }}>\tau\right)$ follows a short-tailed distribution, and mean value and variance can be defined. Our experiments confirm this trend. Figure 1d (squares, circles and diamonds) displays DRs characteristic $P_{\text {off }}\left(\tau_{\text {off }}>\tau\right)$ trends when $\langle N\rangle>1$ for binning times as low as $1 \mathrm{~ms}$ : for samples with thick shell (DR1, DR3 and DR4) we have typically observed values $\mu>2$ and a probability to keep an emission intensity lower than th for periods longer than $50 \mathrm{~ms}$ as low as $P_{\text {off }}\left(\tau_{\text {off }}>50 \mathrm{~ms}\right)=0.001$. As detailed in the SI, thick-shell samples PL is never confused with the noise time trace, independently on the chosen binning time. This is not the case for DRs with standard shell thickness (sample DR2), which show short and long intervals where emission counts superpose to the noise level (Figure 1c and Figure S6 of the SI). This consequently leads to a $\mu$ value lower than 1 (triangles in Figure 1d, see SI for a more detailed description of off periods distribution).

This statistical analysis confirms the significant impact of DR shell thickness in blinking abatement independently on rod length, thoroughly matching the state of art of spherical $\mathrm{CdSe} / \mathrm{CdS}$ NCs. An accurate analysis of DRs time trace distributions shows the presence of a grey PL emission. ${ }^{[25,48]}$ Indeed, the cumulative PL intensity histogram (Figure 1b) of the time trace of Figure 1a is not symmetric, as confirmed by its first order derivative shown in red, which is a direct measurement of the histogram slopes. The lower slope at low count rates indicates the presence of additional emission processes characterized by lower intensity (this is observed at both low and high excitation power, see SI for more experimental data). Nevertheless, the overall emission intensity is highly stable, because the grey emission intensity is very close to the bright state. For this reason, it is hard to define a sharp threshold to distinguish between bright and grey emission, differently from the case of giant shell CdSe/CdS NCs. ${ }^{[25]}$ As described in the $\mathrm{SI}$, the ratio between the average intensity emission of the grey and the bright states $\left\langle\mathrm{I}_{\mathrm{g}}\right\rangle /\left\langle\mathrm{I}_{\mathrm{b}}\right\rangle$ has been roughly estimated to be $\sim 0.67$, which corresponds to the grey state quantum efficiency $Q_{T}$, assuming as unitary the quantum efficiency of the bright state. ${ }^{[25]}$ For comparison, in ref. ${ }^{[25]}$ this ratio has been assessed to be $\sim 0.2$.

As reported by Galland et al., ${ }^{[19]}$ a fingerprint of Auger-nonradiative recombination processes behind grey emission is a shorter decay time compared to the bright state one (see the SI). We have therefore performed time-resolved spectroscopy measurements in order to ascertain if this applies to our DRs. In standard DRs $(t \approx 4 \mathrm{~nm})$ PL decays mono-exponentially with time ${ }^{[37,39,47]}$ (in agreement with the law $\mathrm{I}(\mathrm{t})=\mathrm{Ae}^{-\mathrm{kt}}$, as verified for DR2, see Figure S5 of the SI). However, for thicker DRs we observed a different behavior. A typical decay curve for sample DR1 is reported in Figure 2a: when the pump intensity is below the saturation value, PL follows a bi-exponential decay in agreement with the law $I(t)=A \mathrm{e}^{-\mathrm{k}_{1} \mathrm{t}}+B \mathrm{e}^{-\mathrm{k}_{2} \mathrm{t}}$ with $k_{1}=0.0504 \mathrm{~ns}^{-1}$ and $k_{2}=0.0130 \mathrm{~ns}^{-1}$. Figure $2 \mathrm{~b}$ displays the decay curve for photons contributing uniquely to low or high counts. This analysis confirms a variation of the fitting weights $A$ and $B$, specifically providing the following outcome: $A>>B$ for low photon counts $(\mathrm{A} / \mathrm{B}=5.35)$, whilst $\mathrm{B}>>\mathrm{A}$ for high photon counts $(\mathrm{A} / \mathrm{B}=$ $0.34)$. This trend shows that we can assign $k_{1}$ and $k_{2}$ to grey and bright intensities, respectively, even if they are partially overlapping. These results let us state that DRs PL fluctuations are prevalently due to Auger-mediated processes. In order to confirm this, we have expanded a similar analysis also to blinking nanocrystals (Sample DR2) showing results coherent with the charged nanoparticle models, as detailed in the SI. The radiative $\left(k_{T R}\right)$ and non-radiative $\left(k_{T A}\right)$ recombination rates of the trion configuration can be estimated from equations $\mathrm{k}_{1}=\mathrm{k}_{\mathrm{TR}}+\mathrm{k}_{\mathrm{TA}}$ and $\mathrm{Q}_{\mathrm{T}}=\mathrm{k}_{\mathrm{TR}} /\left[\mathrm{k}_{\mathrm{TR}}+\mathrm{k}_{\mathrm{TA}}\right]$, ${ }^{[25]}$ obtaining $\mathrm{k}_{\mathrm{TR}}=0.0338 \mathrm{~ns}^{-1}$ and $\mathrm{k}_{\mathrm{TA}}=0.0166 \mathrm{~ns}^{-1}$. As $\mathrm{k}_{\mathrm{TR}}$ results larger than $\mathrm{k}_{\mathrm{TA}}$, the radiative trion recombination is the favorite relaxation channel, rationalizing the fact that the grey and bright states PL intensities are close one to each other.

Grey emission is the consequence of an increased ratio $\mathrm{k}_{\mathrm{TR}} / \mathrm{k}_{\mathrm{TA}}$ and, in general, is achievable by decreasing $\mathrm{k}_{\mathrm{TA}}{ }^{[25]}$ or increasing $\mathrm{k}_{\mathrm{TR}}$ by means of cavity or plasmonic resonances. ${ }^{[49]}$ We associate our findings $\left(\mathrm{k}_{\mathrm{TR}}>\mathrm{k}_{\mathrm{TA}}\right)$ to a reduced non-radiative Auger rate by virtue of two predominant phenomena. The first is Auger rate scaling as the inverse of the effective volume occupied by the carriers, ${ }^{[50,51]}$ here induced through shell thickening. The second consists in favoring the formation of a $\mathrm{Cd}$, Se, and $\mathrm{S}$ alloy at core/shell interface thanks to a thicker shell grown at a higher temperature synthesis as compared to standard protocols. This leads to a smooth potential at core/shell interface, known to slow down non-radiative Auger recombination. ${ }^{[10,52,53]}$ A further role in altering DRs Auger rates can be also assigned to charge traps. Indeed, it has been demonstrated that electricfields induced by surface charges can modify carriers confinement potential, ${ }^{[54]}$ that is related to Auger rates. ${ }^{[10,52,53]}$ By increasing shell thickness, surface charges are differently displaced and their influence on non-radiative recombination 
(a)

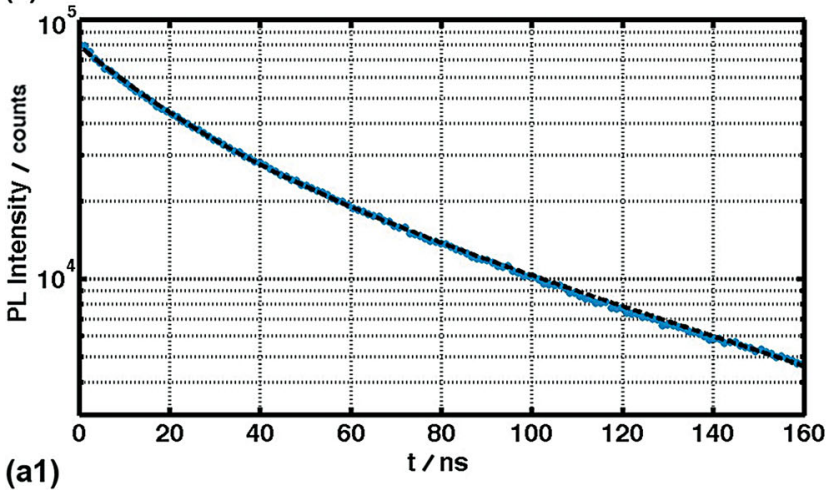

(a1)

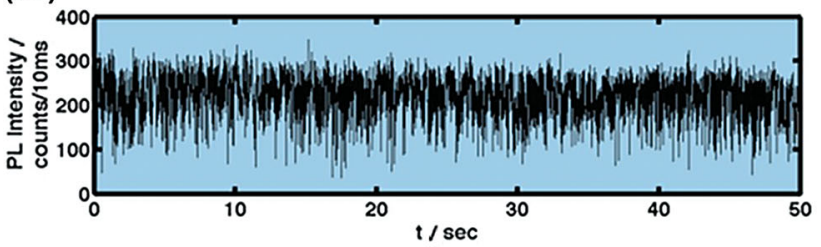

(b)

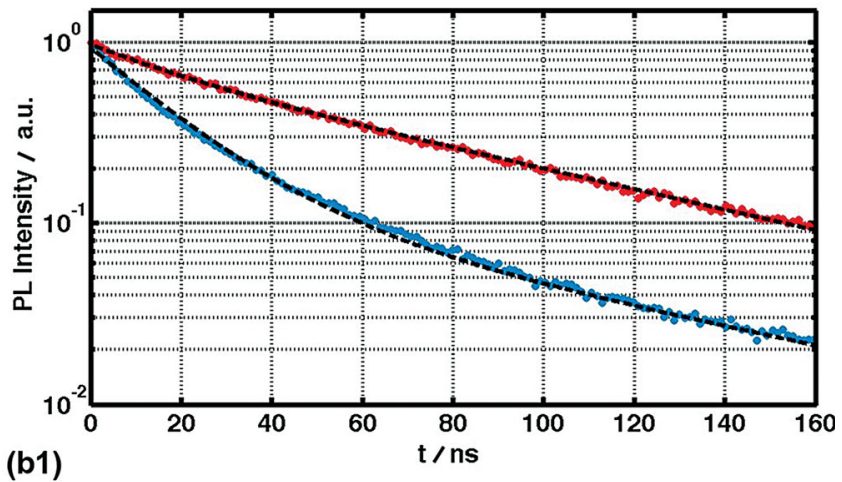

(b1)

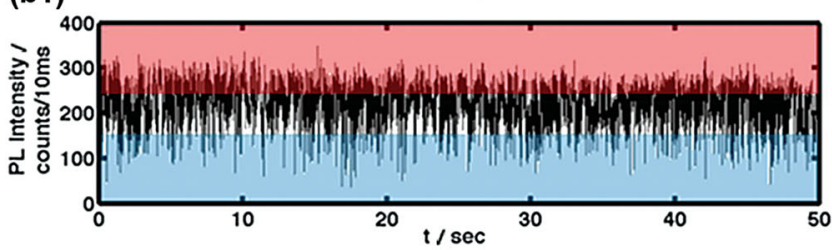

Figure 2. Single DR decay curve. a) Decay curve (blue) and biexponential fitting (black dashed line) for DR1 at low excitation density $(\langle N\rangle \approx 0.6)$. The curve takes into account all the counts collected in the experiment, as evidenced by the light-blue area reported in panel (a1). Best biexponential fitting $I(t)=A e^{-k_{1} t}+B e^{-k_{2} t}$ has been obtained with $k_{1}=0.0504 \mathrm{~ns}^{-1}, k_{2}=0.0130 \mathrm{~ns}^{-1}, A=4.24 \cdot 10^{4}$ and $B=3.70 \cdot 10^{4},(\mathrm{~A} / \mathrm{B} \approx 1.15)$. b) Normalized decay curves for high- (red) and low- (blue) intensity counts of the time trace displayed in panel (b1). Dashed lines represent biexponential fittings. Red dots represent the decay curve for photons which contributed to signals higher than 25000 photons/sec (light-red area in panel (b1)), and have been fitted by: $k_{1}=0.0508 \mathrm{~ns}^{-1}, k_{2}=0.0130 \mathrm{~ns}^{-1}, A=0.2457$, and $B=0.728$, and $\mathrm{A} / \mathrm{B}=0.34$. Blue dots are obtained for photons which contributed to signals lower than 15000 photons/sec (light-blue area in panel (b1)); the corresponding biexponential fitting parameters are: $k_{1}=0.0546 \mathrm{~ns}^{-1}, k_{2}=$ $0.0120 \mathrm{~ns}^{-1}, A=0.7895$, and $B=0.1428$, and $\mathrm{A} / \mathrm{B}=5.35$.

processes can be reduced, since the farther the surface charge from the core, the lower the electric-field influencing the potential at the core/shell interface. The slowing down of non-radiative Auger recombination due to the above-mentioned two predominant effects can be therefore less subject to chargesinduced fluctuations.

Thick-shell DRs are therefore almost always in the on state (here considered as bright plus grey emission), a mandatory condition to produce on-demand photons, as requested for efficient quantum sources. On the other hand, Auger process between two or more e-h pairs is the most efficient strategy to avoid multiple photon emission. In this regard, we have investigated the tendency of shell engineered DRs to emit more than one photon at the same time. We have measured the autocorrelation function of the emitted photons $\mathrm{g}^{(2)}(\tau)=\langle\mathrm{I}(\mathrm{t}) \mathrm{I}(\mathrm{t}-\tau)\rangle /[\langle\mathrm{I}(\mathrm{t})\rangle$ $\langle\mathrm{I}(\mathrm{t}-\tau)\rangle]$ at $\tau=0$ whereby $g^{(2)}(0)$ represents the probability to emit more than one photon per excitation laser pulse. When $g^{(2)}(0)$ approaches zero, bi- and multi-exciton radiative recombinations are limited. ${ }^{[30,31]}$

The typical histograms of photons delays (proportional to $\left.g^{(2)}(\tau)\right)$, namely the coincidence histograms $h(\tau)$, reported in Figure 3a-c for DR2, DR1 and DR3 respectively, show an almost negligible peak at $\tau=0$ for low excitation powers. It is worth noting that it remains very low even for large $\langle N\rangle$ if compared with the peaks at non-zero delay, despite a slight increase as a function of $\langle N\rangle$ is visible. Higher values are instead shown by DR4 both for low and high excitation powers (Figure 3d).

This effect, quantified in terms of average value and standard deviation of $g^{(2)}(0)$ in samples DR2, DR1, DR3 and DR4, is displayed in Figure 3a1-d1, respectively. For non-blinking samples, at $\langle N\rangle \approx 1$, we have found $g^{(2)}(0) \approx 0.075 \pm 0.025, g^{(2)}(0) \approx$ $0.077 \pm 0.041$ and $g^{(2)}(0) \approx 0.22 \pm 0.07$ for DR1, DR3 and DR4, respectively, thus quantifying the single-photon character for these samples. For all samples reported in Figure $3, g^{(2)}(0)$ approaches a close-to-zero constant minimum when $\langle N\rangle \leq 1$, while it raises when $\langle N\rangle$ increases. The number of photons generated by these states depends on the efficiency of the nonradiative Auger recombination between multiexcitons: the slower this Auger process, the higher the probability to emit multiple photons at the same time, resulting in an increased $g^{(2)}(0)$ value for large $\langle N\rangle$ thereof.

It is worth pointing out that the $g^{(2)}(0)$ values at high excitation powers depend on shell length: the longest samples (DR4 and DR3) show maximum values $g^{(2)}(0) \approx 0.47 \pm 0.09$ and $g^{(2)}(0) \approx 0.29 \pm 0.04$, respectively, while for the shortest one (DR1) $g^{(2)}(0) \approx 0.17 \pm 0.04$. On the other hand, as shown for samples DR2 and DR1 the thickness variation does not significantly modify the $g^{(2)}(0)$ value for large $\langle N\rangle$. In our DRs, this behavior can be ascribed to a certain degree of $\Psi_{e}$ delocalization: ${ }^{[9,47,55-57]}$ as sketched in Figure 4a, when rod length is increased from $l_{1}$ to $l_{2}$ the overlap between $\Psi_{\mathrm{e}}$ and $\Psi_{\mathrm{h}}$ decreases, because the average position of the electrons along $x,\langle x\rangle_{e}=\int_{0}^{1} x\left\|\Psi_{e}(x)\right\|^{2} d x$, moves farther from the core, whereas the holes remain localized. This leads to weaker mutual interactions between e-h pairs and thus to smaller $k_{A}$, resulting in a higher value of $g^{(2)}(0)$ for large values of $\langle N\rangle$.

Concerning shell thickness variation, as mentioned above, we suggest that it induces slowering of non-radiative Auger 

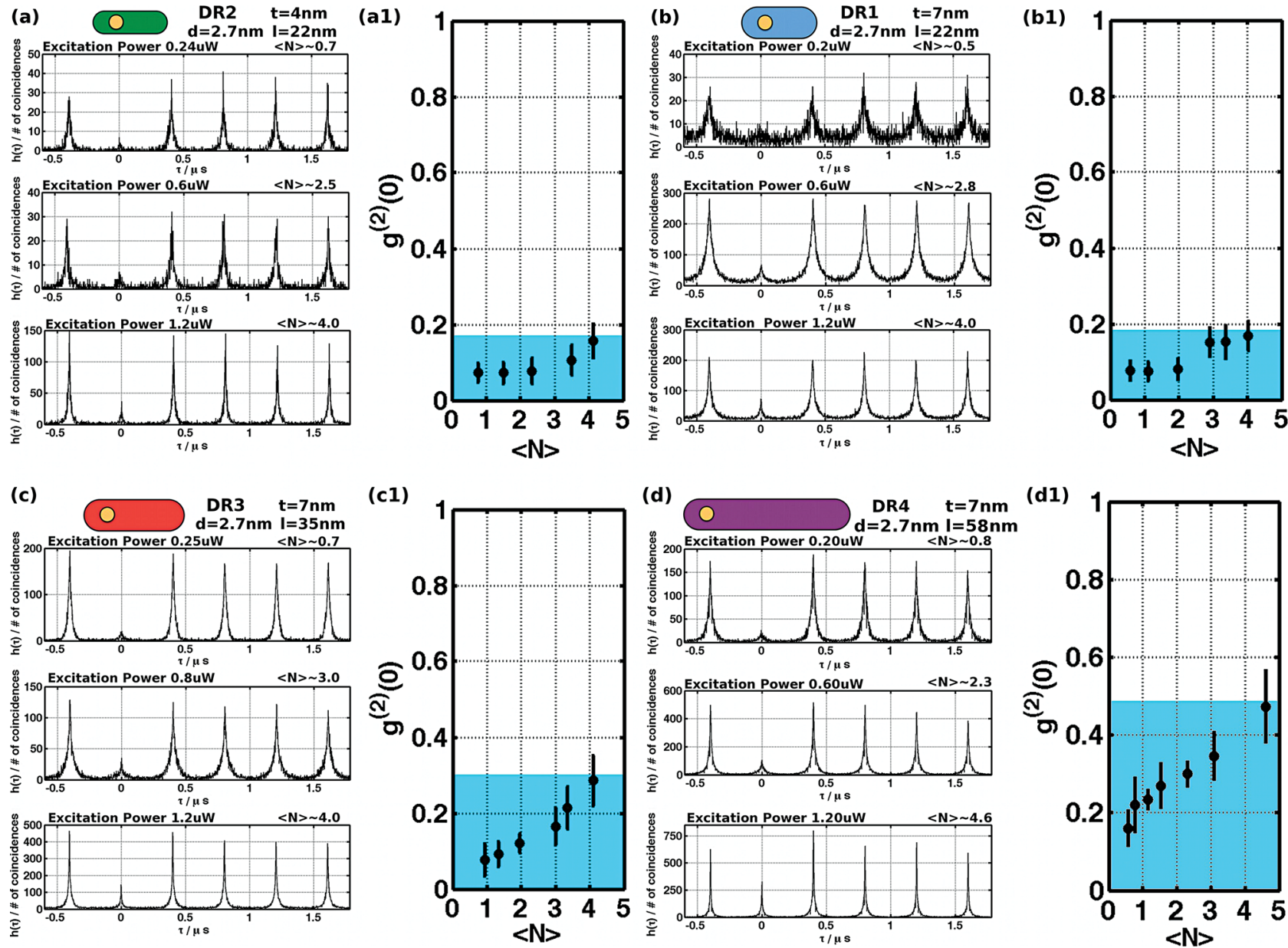

Figure 3. Single-particle autocorrelation measurements. a-d) Coincidences histograms $h(\tau)$ for DR2, DR1, DR3, and DR4, respectively, and for three different excitation densities. $h(\tau)$ is the number of detection events in which two photons have been detected with a delay $\tau$. a1-d1) Values of $g^{(2)}(0)$ as a function of the excitation density for DR2, DR1, DR3, and DR4, respectively (values have been averaged on more than $10 \mathrm{DRs}$ for each sample; error bars are computed as measurements' standard deviation). Light-blue area represents the maximum averaged value of $g^{(2)}(0)$ for large $\langle N\rangle$.

recombination. At the same time, since symmetry along DR transversal directions is preserved, $\Psi_{e}-\Psi_{h}$ spatial overlap is minimally modified, as sketched in Figure $4 \mathrm{~b}$, leading to small variations of $g^{(2)}(0)$. This is confirmed by $g^{(2)}(0)$ measurements obtained for thin DRs (DR2) displayed in Figure 4a, which show values very close to those obtained for sample DR1.

In any case recombination mechanisms in high-temperature grown DRs do not exclusively depend on shell properties. Even if $g^{(2)}(0)$ values obtained for DR1 are already very low considering that also blinking is suppressed, the versatility of quasi-type-II DRs allows to further reduce the multiphoton emission probability. A suitable strategy to achieve that consists in enhancing $\Psi_{\text {e }}$ confinement by increasing core size. ${ }^{[47,56]}$ In this regard, we also investigated a larger core sample (DR5, $d \approx 4.2 \mathrm{~nm}$ ). As expected for very confined structures, lower values of $g^{(2)}(0)$ have been obtained for all the investigated excitation densities (see Figure S7 and SI for more details on sample DR5). Further optimization on shell thickness grown on larger core particles would possibly lead to even more pure non-blinking singlephoton emitting DRs.
In summary, our findings suggest that non-blinking and single-photon emission can be simultaneously obtained in DRs nanocrystals. Moreover, the results presented in this manuscript show that, in the investigated range, a certain degree of independence between the two processes exists, as foreseen in other works. ${ }^{[11,13,26]}$ Indeed we have observed that when DRs shell thickness is increased from $t_{\mathrm{DR} 2} \approx 4 \mathrm{~nm}$ to $t_{\mathrm{DR} 1} \approx 7 \mathrm{~nm}$, keeping $d$ and $l$ unchanged, $\mu$ increases well above 2 , while $g^{(2)}(0)$ is weakly modified. When instead $t$ and $d$ remain constant and $l$ is increased from $l_{\mathrm{DR} 1} \approx 22 \mathrm{~nm}$ to $l_{\mathrm{DR} 3} \approx 35 \mathrm{~nm}$ and $l_{\mathrm{DR} 4} \approx$ $58 \mathrm{~nm}, \mu$ still remains larger than 2 , well above the limit for heavy tailed distributions, giving clear evidence for non-blinking behavior, while $g^{(2)}(0)$ for large $\langle N\rangle$ increases from $0.17 \pm 0.04$ to $0.29 \pm 0.04$ and $0.47 \pm 0.09$. By controlling the DRs shell thickness, it is thus possible to address blinking behavior preserving single-photon emission, whereas, shell elongation in thick DRs preserves non-blinking behavior but modifies the wavefunction of the electrons, influencing multi-photon emission thereof.

Here we have associated these findings to two Auger-mediated energy transfer mechanisms among charges 
(a)

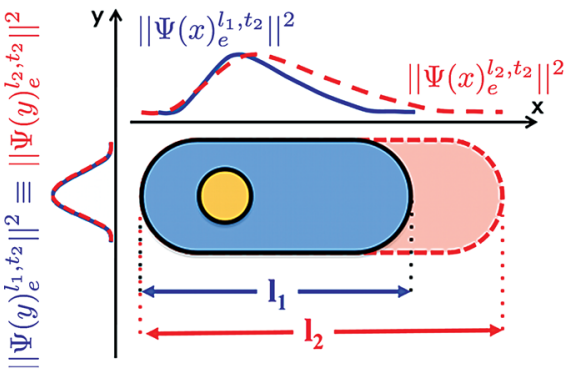

(b)

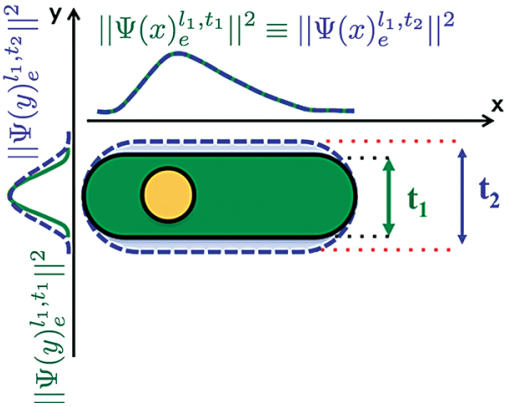

aperture N. A. $=0.95$ for samples DR1 and DR3 and through an oil immersion objective $(\mathrm{N}$. A. = 1.40) for samples DR2, DR4 and DR5. The NC PL emission was collected by the same objective and the obtained image was then delivered into a highsensitivity Hanbury Brown and Twiss setup based on a pair of PerkinElmer fast avalanche photodiodes in order to measure autocorrelation function and decay curves of the detected light.

\section{Supporting Information}

Figure 4. Sketch summarizing the influence of the conformational changes of DRs on the wavefunctions of the electrons. a) When shell length is increased from $I_{1}$ to $I_{2}$ while keeping its thickness constant, the wavefunction of the electrons $\left(\Psi_{\mathrm{e}}\right)$ is also elongated along $x$ and the average spatial position of electrons moves farther from the core. $\Psi_{\mathrm{e}}$ is instead unchanged for both transversal directions $y$ and $z$ for each section of $x$. b) If shell thickness is increased from $t_{1}$ to $t_{2}$ while keeping length fixed, $\Psi_{\mathrm{e}}$ along $x$ is unchanged, while $\Psi_{\mathrm{e}}$ along $y$ and $z$ is broadened, but $\Psi_{\mathrm{e}}-\Psi_{\mathrm{h}}$ overlap is minimally modified because electrons and holes continue to share the same average position. The wavefunction of the holes $\left(\Psi_{h}\right)$ is not reported because it is almost unaffected by shell size and shape engineering.

inside the nanocrystals, since the results reported in Figure 2 and section $\mathrm{V}$ of the SI suggest that also PL blinking is prevalently due to Auger-like phenomena ${ }^{[19,25]}$ and the dependence of DRs photonic properties are consistent with this explanation. However, we should say that literature is controversial on this subject, and the physical interpretation of blinking is being significantly reviewed in very recent works. ${ }^{[17-19]}$ Nevertheless, our experimental evidence demonstrates that in elongated DRs its occurrence can be restricted by shell tailoring and, more importantly, that this does not affect the single-photon emission properties of the NPs. The possibility to obtain non-blinking, singlephoton emission supported by the flexibility of synthesis procedures, lets us envision that these results bring colloidal NCs one step closer to broad applications, paving the way for their implementation as stable and high-intensity room-temperature quantum sources.

\section{Experimental Section}

Synthesis of $\mathrm{CdSe} / \mathrm{CdS}$ dot-in-rods: CdSe/CdS DRs were synthesized using a seeded-growth approach. ${ }^{[15]}$ We here briefly describe the main differences with the protocol described in ref. ${ }^{[15]}$, while an extended procedure is given in the SI. CdSe cores where synthesized accordingly to ref. ${ }^{[15]}$ and the resulting nanoparticles (average diameter $2.7 \mathrm{~nm}$ ) showed band-edge absorption peak at $536 \mathrm{~nm}$. In order to perform the shell growth starting from the $\mathrm{CdSe}$ seeds, a solution of $\mathrm{S}$ precursorTOP-CdSe nanodots is then injected in a flask containing CdO, TOPO, ODPA, HPA and TOP at a temperature of $380^{\circ} \mathrm{C}$ for samples DR 1 and DR3 and $350{ }^{\circ} \mathrm{C}$ for samples DR2, DR4 and DR5 (see Figure S1 of the $\mathrm{SI})$. After the injection, the dark red CdSe/CdS nanocrystal solution is allowed to grow for $20 \mathrm{~min}$ for samples DR1 and DR3, 8 min for samples DR2 and DR5, and $40 \mathrm{~min}$ for sample DR4. The reaction is then stopped by removing the heating mantle and the resulting DRs dispersed in toluene. A detailed synthesis procedure is given in the SI.

Optical measurements: All measurements were performed at RT in air by using a confocal microscope. The whole optical path is described in the SI and sketched in Figure S2 of the SI. Briefly, each single NC was excited by a $2.5 \mathrm{MHz}$ picosecond-pulsed laser diode emitting at $404 \mathrm{~nm}$, focused on the sample through an air objective with numerical
Supporting Information is available from the Wiley Online Library or from the author.

\section{Acknowledgements}

The authors gratefully thank Liberato Manna and Giovanni Morello for fruitful discussions and Mathieu Manceau for the help in the last measurements. Financial support from the French research council ANR, under the project SENOQI, and from the Italian Ministry of Instruction, University and Research (Project "FIRB - Hub di ricerca italo-giapponese sulle nanotecnologie"), are acknowledged. This work was partially supported by PON project "ITEM". F.P., S.V., M.D.V., and A.B. also acknowledge financial support from the Université Franco-Italienne/Università Italo-Francese. A.B. is a member of the Institut Universitaire de France. L.C. and P.D.C. acknowledge financial support from the Italian Ministry of Education, University and Research, through the project AEROCOMP (contract MIUR no. DM48391).

Received: August 2, 2012

Revised: October 29, 2012

Published online:

[1] C. Murray, D. Norris, M. G. Bawendi, J. Am. Chem. Soc. 1993, 115, 8706.

[2] P Michler, A. Imamoglu, M. D. Mason, P. J. Carson, G. F. Strouse, S. K. Buratto, Nature 2000, 406, 968.

[3] T. M. Babinec, B. J. M. Hausmann, M. Khan, Y. Zhang, J. R. Maze, P. R. Hemmer, M. Lončar, Nat. Nanotechnol. 2010, 5, 195.

[4] L. Marseglia, J. Hadden, A. Stanley-Clarke, J. Harrison, B. Patton, Y. L. D. Ho, B. Naydenov, F. Jelezko, J. Meijer, P. Dolan, Appl. Phys. Lett. 2011, 98, 133107.

[5] J. Claudon, J. Bleuse, N. S. Malik, M. Bazin, P. Jaffrennou, N. Gregersen, C. Sauvan, P. Lalanne, J. M. Gérard, Nat. Photonics 2010, 4, 174.

[6] K. Lee, X. Chen, H. Eghlidi, P. Kukura, R. Lettow, A. Renn, V. Sandoghdar, S. Götzinger, Nat. Photonics 2011, 5, 166.

[7] M. Nirmal, B. Dabbousi, M. Bawendi, J. Macklin, J. Trautman, T. Harris, L. Brus, Nature 1996, 383, 802.

[8] Y. Chen, J. Vela, H. Htoon, J. L. Casson, D. J. Werder, D. A. Bussian, V. I. Klimov, J. A. Hollingsworth, J. Am. Chem. Soc. 2008, 130, 5026.

[9] B. Mahler, P. Spinicelli, S. Buil, X. Quélin, J. P. Hermier, B. Dubertret, Nat. Mater. 2008, 7, 659.

[10] X. Wang, X. Ren, K. Kahen, M. A. Hahn, M. Rajeswaran, S. Maccagnano-Zacher, J. Silcox, G. E. Cragg, A. L. Efros, T. D. Krauss, Nature 2009, 459, 686.

[11] Y. S. Park, A. Malko, J. Vela, Y. Chen, Y. Ghosh, F. García-Santamaría, J. Hollingsworth, V. Klimov, H. Htoon, Phys. Rev. Lett. 2011, 106, 187401. 
[12] S. Buil, P. Spinicelli, I. Mallek-Zouari, G. Camps, X. Quélin, B. Mahler, B. Dubertret, J. Hermier, J. Phys. B-At. Mol. Opt. 2009, 42, 114003.

[13] J. Zhao, O. Chen, D. B. Strasfeld, M. G. Bawendi, Nano Lett. 2012, 12, 4477.

[14] D. V. Talapin, R. Koeppe, S. Götzinger, A. Kornowski, J. M. Lupton, A. L. Rogach, O. Benson, J. Feldmann, H. Weller, Nano Lett. 2003, 3, 1677.

[15] L. Carbone, C. Nobile, M. De Giorgi, F. Della Sala, G. Morello, P. Pompa, M. Hytch, E. Snoeck, A. Fiore, I. R. Franchini, Nano Lett. 2007, 7, 2942.

[16] L. Carbone, P. D. Cozzoli, Nano Today 2010, 5, 449.

[17] J. Zhao, G. Nair, B. R. Fisher, M. G. Bawendi, Phys. Rev. Lett. 2010, 104, 157403.

[18] S. Rosen, O. Schwartz, D. Oron, Phys. Rev. Lett. 2010, 104, 157404.

[19] C. Galland, Y. Ghosh, A. Steinbrück, M. Sykora, J. A. Hollingsworth, V. I. Klimov, H. Htoon, Nature 2011, 479, 203.

[20] A. L. Efros, M. Rosen, Phys. Rev. Lett. 1997, 78, 1110.

[21] M. Kuno, D. Fromm, H. Hamann, A. Gallagher, D. Nesbitt, J. Chem. Phys. 2001, 115, 1028.

[22] R. J. Cook, H. Kimble, Phys. Rev. Lett. 1985, 54, 1023.

[23] D. H. Son, J. S. Wittenberg, A. P. Alivisatos, Phys. Rev. Lett. 2004, 92, 127406.

[24] U. Banin, M. Bruchez, A. Alivisatos, T. Ha, S. Weiss, D. Chemla, J. Chem. Phys. 1999, 110, 1195.

[25] P. Spinicelli, S. Buil, X. Quélin, B. Mahler, B. Dubertret, J. P. Hermier, Phys. Rev. Lett. 2009, 102, 136801

[26] C. Galland, Y. Ghosh, A. Steinbrück, J. A. Hollingsworth, H. Htoon, V. I. Klimov, Nature Commun. 2012, 3, 908.

[27] D. Chepic, A. L. Efros, A. Ekimov, M. Ivanov, V. Kharchenko, I. Kudriavtsev, T. Yazeva, J. Lumin. 1990, 47, 113.

[28] J. A. McGuire, J. Joo, J. M. Pietryga, R. D. Schaller, V. I. Klimov, Accounts Chem. Res. 2008, 41, 1810.

[29] F. García-Santamaría, Y. Chen, J. Vela, R. D. Schaller, J. A. Hollingsworth, V. I. Klimov, Nano Lett. 2009, 9, 3482.

[30] B. Fisher, J. M. Caruge, D. Zehnder, M. Bawendi, Phys. Rev. Lett. 2005, 94, 87403.

[31] G. Nair, J. Zhao, M. G. Bawendi, Nano Lett. 2011, 11, 2145.

[32] F. García-Santamaría, S. Brovelli, R. Viswanatha, J. A. Hollingsworth, H. Htoon, S. A. Crooker, V. I. Klimov, Nano Lett. 2011, 11, 687.

[33] H. Htoon, J. Hollingsworth, R. Dickerson, V. Klimov, Phys. Rev. Lett. 2003, 91, 227401

[34] M. Saba, S. Minniberger, F. Quochi, J. Roither, M. Marceddu, A. Gocalinska, M. V. Kovalenko, D. V. Talapin, W. Heiss, A. Mura, Adv. Mater. 2009, 21, 4942.

[35] R. D. Schaller, M. A. Petruska, V. I. Klimov, Appl. Phys. Lett. 2005, 87, 253102.
[36] D. Steiner, D. Dorfs, U. Banin, F. Della Sala, L. Manna, O. Millo, Nano Lett. 2008, 8, 2954.

[37] G. Morello, F. Della Sala, L. Carbone, L. Manna, G. Maruccio, R. Cingolani, M. De Giorgi, Phys. Rev. B 2008, 78, 195313.

[38] F. Pisanello, L. Martiradonna, P. Spinicelli, A. Fiore, J. Hermier, L. Manna, R. Cingolani, E. Giacobino, M. De Vittorio, A. Bramati, Superlattice. Microst. 2010, 47, 165.

[39] F. Pisanello, L. Martiradonna, G. Leménager, P. Spinicelli, A. Fiore, L. Manna, J. P. Hermier, R. Cingolani, E. Giacobino, M. De Vittorio, Appl. Phys. Lett. 2010, 96, 033101.

[40] S. Deka, A. Quarta, M. G. Lupo, A. Falqui, S. Boninelli, C. Giannini, G. Morello, M. De Giorgi, G. Lanzani, C. Spinella, J. Am. Chem. Soc. 2009, 131, 2948.

[41] L. Martiradonna, L. Carbone, A. Tandaechanurat, M. Kitamura S. Iwamoto, L. Manna, M. De Vittorio, R. Cingolani, Y. Arakawa, Nano Lett. 2008, 8, 260.

[42] A. Qualtieri, L. Martiradonna, T. Stomeo, M. T. Todaro, R. Cingolani, M. D. Vittorio, Microelectron. Eng. 2009, 86, 1127.

[43] M. De Vittorio, F. Pisanello, L. Martiradonna, A. Qualtieri, T. Stomeo, A. Bramati, R. Cingolani, Opto-electron. Rev. 2010, 18, 1.

[44] A. Qualtieri, F. Pisanello, M. Grande, T. Stomeo, L. Martiradonna, G. Epifani, A. Fiore, A. Passaseo, M. De Vittorio, Microelectron. Eng. 2010, 87, 1435.

[45] F. Pisanello, A. Qualtieri, T. Stomeo, L. Martiradonna, R. Cingolani, A. Bramati, M. De Vittorio, Opt. Lett. 2010, 35, 1509.

[46] R. Krahne, G. Morello, A. Figuerola, C. George, S. Deka, L. Manna, Phys. Rep. 2011, 501, 75.

[47] G. Rainó, T. Stöferle, I. Moreels, R. Gomes, J. S. Kamal, Z. Hens, R. F. Mahrt, ACS Nano 2011, 5, 4031

[48] A. V. Malko, Y. S. Park, S. Sampat, C. Galland, J. Vela, Y. Chen J. A. Hollingsworth, V. I. Klimov, H. Htoon, Nano Lett. 2011, 11, 5213.

[49] X. Ma, H. Tan, T. Kipp, A. Mews, Nano Lett. 2010, 10, 4166.

[50] V. I. Klimov, A. A. Mikhailovsky, D. W. McBranch, C. A. Leatherdale, M. G. Bawendi, Science 2000, 287, 1011.

[51] I. Robel, R. Gresback, U. Kortshagen, R. D. Schaller, V. I. Klimov, Phys. Rev. Lett. 2009, 102, 177404.

[52] G. E. Gragg, A. L. Efros, Nano Lett. 2010, 10, 313.

[53] J. I. Climente, J. L. Movilla, J. Planelles, J. Appl. Phys. 2012, 111, 043509.

[54] J. Müller, J. M. Lupton, A. L. Rogach, J. Feldmann et al., Phys. Rev. B 2005, 72, 205339.

[55] J. Müller, J. Lupton, P. Lagoudakis, F. Schindler, R. Koeppe, A. Rogach, J. Feldmann, D. Talapin, H. Weller, Nano Lett. 2005, 5, 2044.

[56] A. Sitt, F. D. Sala, G. Menagen, U. Banin, Nano Lett. 2009, 9, 3470.

[57] M. Zavelani-Rossi, M. G. Lupo, F. Tassone, L. Manna, G. Lanzani, Nano Lett. 2010, 10, 3142. 